\title{
Wie steht es um die urologische Versorgungsforschung?
}

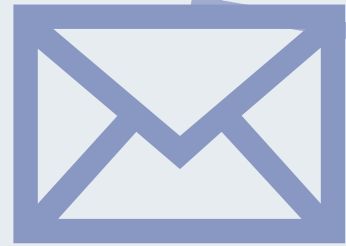

\author{
In URO-NEWS 9/2011 hat Prof. Lothar Weißbach den Zustand der \\ urologischen Versorgungsforschung kritisiert. Dr. Götz Geiges, \\ Vorstandsvorsitzender des IQUO, bezieht dazu Stellung.
}

$\mathrm{D}$ ie Diskussion um den Stellenwert der Versorgungsforschung in Deutschland zeigt zum einen die Bedeutung des Themas, zum anderen wird auch deutlich, dass den Worten mancher Protagonisten nicht immer Taten folgen.

Es stellt sich die Frage, welche Akteure an welcher Stelle eingebunden sind. Der Interessenverband zur Qualitätssicherung der Arbeit niedergelassener Uro-Onkologen in Deutschland (IQUO e.V.) hat frühzeitig die Notwendigkeit erkannt, Qualitätsdaten zur Versorgung in der täglichen Praxis standardisiert zu erheben. Das Deutsche Netzwerk Versorgungsforschung (DNVF) steht für die derzeitigen anerkannten Standards dieses Forschungsgebietes. Der IQUO ist als einzige urologische Vertretung Mitglied im DNVF. Ziel ist, die Standards im Bereich der Urologie anzupassen und umzusetzen.

Eine aktive Mitarbeit im DNVF basiert ausschließlich auf dem Ergebnis einer qualitativ hochwertigen und zielorientierten Arbeit, einer überzeugenden Struktur, Organisation und Dokumentation von Qualität in der Praxis. Mit gut 350 IQUO-
Mitgliedern bilden wir die kompletten Behandlungsverläufe von 19.631 Prostatakarzinom-, 4.964 Urothelkarzinom-, 1.445 Nierenzellkarzinom- sowie rund $100 \mathrm{Pe}$ niskarzinom-Patienten ab. Somit verfügt der IQUO über valide Daten von gut $95 \%$ der urologischen Tumoren. In Kürze wird auch die Entwicklung zur Dokumentation des Hodenkarzinoms abgeschlossen sein. Diese wissenschaftliche Dokumentation aller Diagnostik- und Behandlungsleistungen in der urologischen Praxis ist unser Beitrag zur Versorgungsforschung. Damit ist möglich, viele Fakten über Demografie, Morbidität, Behandlungsrealität etc. wissenschaftlich abzubilden. Wir haben einen Standard etabliert, der durch das besondere Augenmerk auf die Qualität der Verbandsdaten fortlaufend überprüft und verbessert wird. Automatische Queries, verbandseigenes Inhouse- und Vor-OrtMonitoring, permanente Schulungen sowie Fort- und Weiterbildungen stellen die Qualität sicher. Um eine erfolgreiche Versorgungsforschungsarbeit leisten zu können, bedarf es natürlich einer Kooperation mit allen handelnden Akteuren im medi- zinischen Bereich, die transparent und kodexkonform verläuft. Wir laden alle ein, mit uns in einen konstruktiven Dialog zu treten, die Interesse an der Qualitätsssicherung und -verbesserung der uro-onkologischen Diagnostik und Therapie haben.

Keine Phase-I- bis -IV-Studie fände ohne die Industrie-Kooperation statt. Gerade für eine qualitativ hochwertige Behandlung und Versorgung schwerkranker Patienten ist eine Kooperation mit allen am Gesundheitssystem Beteiligten unabdingbar. Nur so können Sinnhaftigkeit, Qualität, Therapieerfolg sowie entsprechende Konsequenzen und Handlungsempfehlungen abgebildet und abgeleitet werden. Insofern streben wir eine weitere Verzahnung mit anderen wissenschaftlichen Einrichtungen, Kliniken und Fachgesellschaften an. Ziel ist, sich unseres Datenpools zu bedienen und miteinander an der permanenten Verbesserung der urologischen Praxis zu arbeiten. Wir sollten uns alle gemeinsam von Zeit zu Zeit vergegenwärtigen, was unser Schwerpunkt ist: die effiziente und qualitativ hochwertige Behandlung unserer Patienten in der urologischen Praxis und nicht die Durchsetzung von Partikularinteressen.

Dr. Götz Geiges, Berlin

Die Redaktion behält sich vor, Leserbriefe in gekürzter Form zu veröffentlichen. 